tubes wound in parallel. The filament leads are threaded through the bore of one of the tubes and the grid-driving potential is supplied through an inner conductor of the other. At the filament end of the coil, each side of the filament is connected to the tubes via a condenser, the other end of the coil being earthed. Thus the filament current and griddriving voltage are delivered at the required location with the driver and filament transformers maintained at earth potential. The article describes and illustrates the apparatus in some detail.

\section{ISO-MAGNETIC CHARTS OF THE GLOBE}

$I^{\mathrm{N}}$

a series of articles which have appeared in issues of the quarterly journal Terrestrial Magnetism and Atmospheric Electricity (commencing with the issue dated December, 1940), Prof. Sydney Chapman gives the results of his valuable investigation of the general form which iso-magnetic curves should take on magnetic charts of the globe, and of the interrelation between the curves for certain components of the earth's magnetic field.

The magnetism of the globe assumed in the firstnamed investigation is that due to a 'dipole' centred upon an axis of the earth. Although this simplification only roughly corresponds to observed facts, and although in a generalized discussion local irregularities of the earth's field cannot, of course, be taken into account, some interesting and instructive geometrical features are shown to exist, knowledge of which can be applied with advantage in the construction of magnetic charts. Examples are: the shape of the curves near their nodes and singular points, the correspondence and relation of nodes and foci, and the course of the iso-lines near the magnetic (or dip) poles.

In the second branch of his investigation, Prof. Chapman develops and demonstrates a method of testing the mutual consistency of data derived from iso-magnetic charts of declination and horizontal intensity. Normally, the charts are produced independently. The line-integral of the magnetic force round a closed contour on the earth's surface can be used to calculate the intensity of current electricity passing between atmosphere and earth within the boundary. Direct measurement of air-earth current, however, shows that the current is actually much too small to be effective on the scale of the charts; and if significant values are obtained when the line-integral is taken, derived from chart readings of declination and horizontal intensity, round a vanishingly small area, the inference to be drawn is that errors exist in the data. These may originate in either of the charts or in both, and will probably be due to actual errors in the placing of the iso-lines by the chart constructor. In this case the position of such lines may be improved by trial even in the absence of fresh data ; and since horizontal intensity is in general less well determined than declination, substantial improvement in charts of horizontal intensity may be possible by this means.

Prof. Chapman has recently extended his investigation to isopors representing secular change in the values of the elements, a problem of considerably greater complexity. The results are published as Notes VII and VIII of the series, in the issue of Terrestrial Magnetism of June, 1942.
Isoporic curves, more particularly over the oceans, are to a large extent inferential, since with the sources at present in existence for procuring observational data they are of necessity based on unevenly scattered and relatively scanty information, very largely obtained from fixed land observatories.

Secular change in the earth's magnetic field is itself subject to change which is sometimes quite rapid in limited areas. Consequently, a complete picture of the changes occurring over the whole globe at any one time must always be an ideal attainable only in retrospect.

The lack of mutual consistency detected by Prof. Chapman in areas on the charts he has examined emphasizes the need of additional observations of secular change in particular regions of the globe. W. M. W ITCHELI.

\section{PUTREFACTION IN CATTLE HIDES}

$T$

$\mathrm{HE}$ best method of drying hides is to lace them flat in a frame and dry them with access of air to both sides. Some delay between flaying and the suspension of the hide for drying is inevitable. Commercial practice has allowed this delay to extend to several hours, sometimes to 1-2 days. Experiments were therefore carried out at Kano in Nigeria and at Isiolo and Mombasa in Kenya ("Results of Delayed Drying Trials in Nigeria and Kenya", Bull. Imperial Institute, 40, No. 2; 1942). These three places represent a series of conditions of increasing atmospheric humidity and, to a less degree, increasing mean shade temperature, Kano having considerably the driest and slightly the coolest mean shade temperature and Mombasa the wettest and warmest.

In the experiment at Kano the hides were divided into two lots; one lot was given an arsenical dip immediately after flaying, the other lot was given an arsenical dip at the end of the delay period. Arsenical dips, if sufficiently strong, have some bactericidal effect. During the delay period the hides were folded hair out and left in the sun. Drying was in the sun. The dried hides were shipped to England and tanned for sole leather. The hides arsenicated before delay from the 8-and 16-hour periods showed no grain damage. Hides arsenicated before delay but left for longer periods and hides arsenicated after delay all showed slight grain damage in the finished leather. Except for this superficial damage the hides all proved to be sound. There was no damage due to Dermestes beetles.

In the experiments at Isiolo and Mombasa the experimental hides were divided in the line of the backbone, one side suspended for drying within two hours of flaying, the other folded and put in the sun for delay periods up to thirty-two hours. Drying was in the sun. In these hides there was a striking difference between the control and those with delayed drying. All the control half-hides were perfectly sound; the delay half-hides showed varying degrees of putrefaction, worse in the Mombasa hides than the Isiolo hides and increasing with the length of delay. All the control hides were free from damage by Dermestes beetles; the delay hides showed considerable damage both on the grain and in the carium, increasing with the degree of putrefaction. It should be noted that delay and control half-hides were shipped in the same bale. Although considerable putrefaction occurred in $\operatorname{som} \theta$ cases it is 